\title{
Contribution of $\vec{E} \times \vec{B}$ drifts and parallel currents to the divertor asymmetries
}

\author{
V. Rozhansky ${ }^{1}$, P. Molchanov ${ }^{1}$, I. Veselova ${ }^{1}$, S. Voskoboynikov ${ }^{1}$, A. Kirk ${ }^{2}$, D. Coster ${ }^{3}$ \\ ${ }^{1}$ St.Petersburg State Polytechnical University, Polytechnicheskaya 29, 195251 St.Petersburg, Russia \\ ${ }^{2}$ EURATOM/CCFE Fusion Association, Culham Science Centre, Abingdon, Oxon, OX14 3DB, UK \\ ${ }^{3}$ Max-Planck Institut für Plasmaphysik, EURATOM Association, D-85748 Garching, Germany
}

\section{Introduction}

The role of $\vec{E} \times \vec{B}$ drifts and parallel currents in the formation of the parameters in a divertor of a tokamak was discussed in several publications, see e.g. [1]-[5]. One possibility is that the radial $\vec{E} \times \vec{B}$ drifts play the main role due to the radial flows from low field side (LFS) divertor to the HFS one (for single null divertor configuration). Other group of authors considered the poloidal $\vec{E} \times \vec{B}$ drifts as a main source of additional divertor asymmetries. Several simulations with 2D transport codes were not able to give unambiguous answer to this problem since the role of the drifts was masked by the usual LFS - HFS divertor asymmetry caused by the geometry. The parallel current flowing in the divertor adds to the complexity of the problem.

In this paper a systematical study of these effects is reported on the basis of the analysis of the simulations performed by B2SOLPS5.2 transport code. To separate the asymmetry caused by geometry a connected double-null configuration of MAST was chosen which has up-down symmetry so that all up-down asymmetries should be connected with $\vec{E} \times \vec{B}$ drifts and parallel currents. It is demonstrated that the asymmetry is caused by the poloidal $\vec{E} \times \vec{B}$ drift while the role of the radial drifts is insignificant.

The main physical effect is connected with the change of the parallel particle fluxes in the recycling zone in the presence of the $\vec{E} \times \vec{B}$ drifts and corresponding changes of the pressure distribution to provide the required parallel fluxes. The larger plasma density is observed at the divertor where the $\vec{E} \times \vec{B}$ drift is directed away from the plate while the plate where the $\vec{E} \times \vec{B}$ drift is directed towards the plate has lower density. The divertor with higher density has lower temperature and as a consequence the temperature asymmetry cases thermal current from higher temperature divertor to that with a lower temperature. The convective electron heat flow has the opposite direction, i.e. is directed from the colder and denser divertor towards the hotter and lower dense one. As a result the power asymmetry is also observed. 
For the single null configuration the LFS-HFS asymmetry caused by geometry causes a thermal current from the hotter LFS divertor to the colder and denser HFS one. Its contribution to the divertor's asymmetry is investigated for the ITER-like parameters. It is demonstrated that the convective electron heat flux from the HFS to the LFS divertor amplifies the asymmetry with respect to the simulations with parallel current switched off. Switching on the $\vec{E} \times \vec{B}$ drifts causes further amplification of the asymmetry.

\section{Simulations of MAST connected double null (CDN) discharges}

Three H-mode discharges in the CDN configuration with $\nabla B$ drift directed towards the $X$-point were chosen for simulation. The simulations were performed with the B2SOPLS5.2 transport code with the fluid description of neutrals. All shots were simulated both with all drifts and currents switched on and off. In the variants without drifts and currents the plasma parameters should have an up-down symmetry since the basic equations, boundary conditions and simulation grid in the absence of drifts and currents provide this symmetry. In reality the updown symmetry is not ideal due to the non-ideal grid.

The typical density, electron temperature and electrostatic potential distributions for shot №17469 without gas puff are shown in Fig.1. The following transport coefficients were chosen: diffusion coefficient $D=2.0 \mathrm{~m}^{2} / \mathrm{s}$, electron and ion heat conductivity coefficients $\kappa_{e} / n_{e}=2.4 m^{2} / s, \kappa_{i} / n_{e}=2.4 m^{2} / s$. The anomalous diffusion coefficient was reduced by a factor of 10 and the electron and ion heat conductivities were reduced by a factor of 2 in the transport barrier region $(2 \mathrm{~cm}$ inside the separatrix and $0.05 \mathrm{~cm}$ outside the separatrix at the outer mid-plane) similar to other simulations performed for MAST [6]. An up-down asymmetry is clearly seen in the simulations (see also Table 2).

Table 1-3 shows the results of the simulations for the three shots. The numbers are simulations with the drifts and currents included while the numbers in brackets are without the drifts and currents. The abbreviation OU and OL correspond to the outer upper and outer low plates correspondingly, while IU and IL represent inner upper and inner low plates. The maximal values are presented which are at positions at the plates close to the strike points. Note, however, that for the different quantities maxima might be reached at different points. For the potential the separatrix values are shown. In the brackets the results of the simulations without drifts and currents are given. Here $p_{p l}$ is total pressure of electrons and ions, $V_{x}^{E x B}$ is an absolute value of a poloidal $\vec{E} \times \vec{B}$ velocity, $V_{\|}$is an absolute value of a parallel velocity $c_{s} b_{x}$ is a poloidal sound speed. 
One can see that in all three cases the density and temperatures at the plates are approximately symmetrical in the simulations without drifts and currents, the difference might be attributed to the non-ideal simulation grid. When all drifts and currents are switched on the updown symmetry is broken. At the LFS plates the plasma density at the upper plate becomes larger than the density at the lower plate. In contrast at the HFS plates the situation is reversedthe plasma density at the upper plate is smaller than the density at the lower plate. The temperatures up-down asymmetry is opposite to the density asymmetry-the denser divertor correspond to the lower temperature and vise versa. The saturation current to the plates is larger for the plate with higher density.

Since the plasma potential in the SOL decreases from the separatrix in the radial direction, the poloidal $\vec{E} \times \vec{B}$ drift at LFS is directed away from the plate at the upper plate and towards the plate at the lower plate. For the HFS the poloidal drift is directed away from the plate at the lower plate and towards the plate at the upper plate. So we can conclude that the higher density corresponds to the case when the poloidal $\vec{E} \times \vec{B}$ drift is directed away from the plate and lower density corresponds to the situation when the plasma is drifting towards the plate.

From the last two columns of the tables one can see that the poloidal plasma flow associated with poloidal $\vec{E} \times \vec{B}$ drifts $n V_{x}^{E x B}$ is of the same order as the total ion flux to the plate $n c_{s} b_{x}$ with the poloidal sound speed velocity. Since at the plates the following boundary condition is satisfied

$$
n V_{x}^{E x B}+n b_{x} V_{\|}=n c_{s} b_{x},
$$

in the presence of drifts the parallel velocity should adjust itself so that Eq.(1) is satisfied. As a result the parallel velocity at the LFS is larger at the upper plate than at the lower one. For the HFS the situation is reversed.

An example of the poloidal profiles of the parallel velocity in the SOL close to the separatrix at the LFS are shown in Fig.2 for shot №13018. The sign corresponds to the direction towards the plates. In all three simulations the parallel velocity is directed towards the plates, however in principle it might be directed away from the plate provided the large enough $\vec{E} \times \vec{B}$ drift is directed to the plate.

The change in the parallel velocity caused by the drifts requires the corresponding pressure gradient to accelerate or decelerate plasma to or away from the plates. An example of corresponding poloidal density distribution at the LFS plates is shown in Fig.3. At the upper 
plate the density is decreasing towards the plate to provide plasma acceleration towards the plate to compensate the $\vec{E} \times \vec{B}$ drift away from the plate. In contrast, at the lower plate the plasma density is decreasing from the plate towards the $X$-point. As a result the upper divertor has larger density than the lower one. At the HFS the situation is reversed and the density in the upper divertor is larger than in the lower one. This tendency is typical for all shots simulated with the exception of the HFS divertors №13018, where the density itself is very large and the up-down asymmetry is modest.

If the temperature at the divertors is significantly lower than the upstream separatrix temperature the conductive electron heat fluxes to upper and lower plates are almost the same since they are density independent. Therefore the temperatures at the plates should behave as $T_{e} \sim n^{-2 / 3}$ to transfer the heat flow coming from upstream to the walls. Such behavior is seen for two shots, Table.2-3. For shot №13018 the temperatures at the LFS plates are not sufficiently low and the conductive flow to the upper plate (with lower temperature) is larger than to the lower plate.

Since at the hotter plate the floating potential drop is larger than at the colder one a thermal current arises flowing from the hotter to the colder divertor [7]. At the LFS the thermal current flows upward while at the HFS it flows downward as shown in Fig. 4. Note that the thermal currents are modulated by Pfirsch-Schluter (PS) currents (not shown in Fig.4), for further details see [8]. The thermal current is amplified by the PS currents at the LFS equatorial mid-plane and is reduced by the PS current at the HFS mid-plane. To organize the thermal parallel current according to the sheath current-voltage characteristic the potential at the hotter plates should be smaller than the floating potential while at the colder plates the potential should be larger than the floating one. The dimensionless potential $e \varphi / T_{e}$ in the Tables 1-3 is indeed smaller at the hotter plates, lower for the LFS and upper for the HFS, than at the colder ones, upper for the LFS and lower for the HFS (note that presented are the values calculated two cells away from the plate, not exactly at the sheath entrance).

The electron poloidal heat flow associated with the parallel current consists of two parts [9]:

$$
q_{j x}=q_{j x}^{c o n v}+q_{j x}^{\vec{u}}=-3 / 2 b_{x} j_{x} T / e-0.71 b_{x} j_{x} T / e=-2.21 b_{x} j_{x} T / e .
$$

The first contribution is a pure convective heat flow (in the heat balance equation, in the energy balance equation the coefficient would be $5 / 2$ ). The second term is caused by the distortion of the distribution function in the presence of the current. The sum is a total convective heat flux 
associated with the current. The total convective flux is added to the conductive one. In most cases when the divertor temperatures are low enough with respect to the separatrix electron temperature the convective heat flux Eq. (2) is directed downwards at the LFS and upwards at the HFS. This is seen in the power asymmetry in the Tables 2-3. For shot № 13018 the opposite asymmetry in the conductive flux is more important. In general the parallel current amplifies the asymmetry caused by the drifts.

In the MAST experiments due to the uncertainty in the saturation currents and electron temperature measurements it is difficult to conclude on the experimentally observed asymmetry of these parameters. The example of the comparison between the simulations and experimental data is shown in Figs.5,6 for shot №17469. The simulations also contain uncertainty due to the fluid description of neutral particles. This is illustrated by simulation with the kinetic description of neutrals by EIRENE code which was made for this particular shot, Figs.5-6. The difference in the calculated integrated $D_{\alpha}$ signals is modest. In the experiments the integrated $D_{\alpha}$ signal in a similar shot for the low divertor is about 1.5 larger than for the upper one. This might be connected with approximate character of the description of neutrals in the simulations with fluid neutrals.

The power up-down asymmetry obtained in the simulations (Tables 2-3) is consistent with MAST observations [10]-[11], where more power went to the low divertor than to the upper one in various CND shots.

\section{Simulations of ITER L-mode}

To investigate the role of the parallel current and $\vec{E} \times \vec{B}$ drifts in the situation with single-null asymmetric divertor three ITER L-mode shots were simulated by the transport code B2SOLPS5.2. The following transport coefficients were chosen: $\chi_{e}=\chi_{i}=1 \mathrm{~m}^{2} / \mathrm{s}, D_{\perp}=0.3 \mathrm{~m}^{2} / \mathrm{s}$. These transport coefficients coincide with those used in the previous simulations of ITER [12]. Three values of plasma density at the inner side of the simulation domain were chosen: $n=3.75 \cdot 10^{19} ; 5 \cdot 10^{19} ; 7 \cdot 10^{19} \mathrm{~m}^{-3}$. Corresponding values of electron and ion temperatures were chosen in such a way to provide an acceptable heating power $P \approx 90 \div 95 \mathrm{MW}$ : $T_{e}=T_{i}=1.2 ; 0.9 ; 0.7 \mathrm{keV}$. For each scenario three types of simulations were performed. In the first run all electric fields and currents were switched off which corresponds to the simulations performed earlier with B2SOLPS4.3 (curves labeled by ' 1 ' in figures below). The second variant corresponds to so called 'no drift case', when $\vec{E} \times \vec{B}$ and diamagnetic drifts were switched off and hence Pfirsch-Schlueter currents were absent, but the rest parallel current in the SOL and 
sheath potential drops were calculated (curves labeled by ' 2 ' in figures below). This was done to investigate the role of the parallel thermal current in the SOL. In the third type of simulations all terms with electric fields and currents were switched on (curves labeled by ' 3 ' in figures below). For the first scenario, $n=3.75 \cdot 10^{19} \mathrm{~m}^{-3} ; T_{e}=T_{i}=1.2 \mathrm{keV}$, an additional type of simulation was performed which corresponds to the multi-fluid model (all other runs were done for pure deuterium). For this case $C$ and $H e$ were chosen as impurities. At the inner boundary of simulation domain $\mathrm{He}^{+2}$ density of $6.8 \cdot 10^{17} \mathrm{~m}^{-3}$ was taken. Carbon source was determined by the chemical sputtering at the material surface with the sputtering coefficient $\gamma=1 \%$. Deuterium plasma parameters were taken the same as for the single fluid regime. The anomalous values of diffusion and heat conductivity coefficients were chosen equal for all species. Electric fields and currents were switched on in this case. This run is labeled by ' 4 ' on figures below.

Switching on the parallel current in the SOL makes the divertor plasma more asymmetric due to the additional electron heat flow from the inner to the outer divertor, Eq. (2). The contribution of the latter flow could be estimated from Fig.7, where the poloidal electron heat flows with and without convective term between two flux surfaces are plotted. One can see that the heat flow to the inner divertor with account of the parallel current is three times smaller than that without the current.

The density and temperature distributions along the plates are shown in Figs. 8-9. The electron temperature at the inner divertor is more than three times smaller than in the absence of the parallel current. A similar change in the ion temperature is observed. The electron density is changed in the opposite direction and is more than twice larger in the case when the current is switched on. Some increase in the separatrix density is also observed. At the outer divertor the difference between two cases is more modest (of the order of $20 \%$ for the temperatures) due to the smaller difference in the heat flows with and without current coming to the outer divertor. Switching on drifts further amplify in-out asymmetry. It can be also concluded that the presence of impurities doesn't affect much the background plasma parameters (curve '4').

In the medium density case similar effects are observed, Figs. 10-11. In the high density case the change in the divertor parameters is rather modest, even at the inner divertor, Fig. 12.

\section{Discussion}

\subsection{Why the poloidal $\vec{E} \times \vec{B}$ drifts contribute to the divertor asymmetry}


From the simulations made both for connected double null and single null configurations we see that the plasma density increases when the poloidal $\vec{E} \times \vec{B}$ drift is directed away from the plate while the poloidal $\vec{E} \times \vec{B}$ drift towards the plate is responsible for a reduction in density. The temperatures at the divertors vary in the opposite directions. The main reason for this is the change of the parallel (poloidal) pressure gradient in the high recycling zone in the presence of the drifts. Indeed if one neglects the perpendicular transport of momentum in a simplified 1D model in a Cartesian geometry with $x$ being a poloidal co-ordinate, Fig. 13, the parallel momentum balance reads

$$
m_{i} \frac{\partial}{\partial x} n V_{x} V_{\|}=-b_{x} \frac{\partial p}{\partial x}
$$

where $V_{x}=b_{x} V_{\|}+V_{x}^{E x B}$. From Eq. (3) it follows

$$
n V_{x}\left(V_{x}-V_{x}^{E x B}\right)+n b_{x}^{2} c_{s}^{2}=\operatorname{const}(x) .
$$

At the left plate the poloidal velocity is negative and is equal to the poloidal sound speed so the first term in the 1.h.s. is added to the second one. If, for example, $V_{x}^{E x B}=b_{x} c_{s}$, the constant is equal to const $=3 n_{-} b_{x}^{2} c_{s}^{2}$. To the right of the high recycling zone, where the main ionization sources are located, the poloidal velocity becomes smaller and the first term on the r.h.s. of Eq. (3) could be neglected, hence const $=n b_{x}^{2} c_{s}^{2}$. So in this example the pressure at the outer part of the high recycling zone is three times larger than at the plate to organize poloidal flow to the plates. The parallel velocity at the left plate in this example is $V_{\|}=2 c_{s}$. The typical density profile is shown in Fig.11. The large density in the high recycling zone produce larger ionization and accordingly larger particle plow to the plates, hence density at the plate should be large with respect to no drift case.

At the right plate the poloidal velocity is positive and the first term on the r.h.s. of Eq.(4) is smaller than at the left plate. In our example $V_{x}^{E x B}=b_{x} c_{s}$ the parallel velocity is zero at the right plate and const $=n_{+} b_{x}^{2} c_{s}^{2}$. The maximal pressure in the high recycling zone corresponds to $V_{x}(x)=(1 / 2) V_{x}^{E x B}$ and const $=(3 / 4) n b_{x}^{2} c_{s}^{2}$, so the maximal pressure is only $4 / 3$ times larger than the pressure at the plate. The density rise from the plate is more modest than at the left divertor and as a result the density at the right divertor and the particle flux to the right plate are smaller. 
The steep density drop to one plate and almost no density drop towards the other plate, Fig. 3, is consistent with this analysis. Also the behavior of the parallel velocity -acceleration towards one plate and deceleration towards the other one, Fig.2, is similar to what one would expect from above arguments.

\subsection{Why the parallel current contribute to the divertor asymmetry}

Let us consider a simple analytical model for the thermal current taking into account finite parallel conductivity (in [7] an infinite parallel conductivity has been assumed). The PfirschSchlueter currents are not considered in this Section. Let us assume that the right hand divertor is significantly hotter than the other. The equal electron and ion temperatures and constant pressure $n T=\operatorname{const}(x)$ are assumed, which corresponds to $V_{x}^{E x B}$ smaller than the poloidal sound speed, in contrast to the previous section. The poloidal current is given by

$$
j_{x}=j_{\|} b_{x}=\sigma_{\|} b_{x}^{2}\left[\frac{1}{e n} \frac{\partial(n T)}{\partial x}+\frac{0.71}{e} \frac{\partial T}{\partial x}-\frac{\partial \varphi}{\partial x}\right] .
$$

Since the pressure is assumed to be constant and $j_{x}$ is conserved, taking into account that $\sigma_{\|} \sim T^{3 / 2}$, we have for the potential

$$
\varphi=0.71 T / e+A \widetilde{x}+B
$$

The new coordinate is defined as

$$
d \widetilde{x}=d x \frac{T_{+}^{3 / 2}}{T^{3 / 2}},
$$

where $T_{+}$is the temperature at the right hotter plate. In the more complicated case when $b_{x}$ depends on the poloidal coordinate and there is a flux expansion, these effects can be easily taken into account by redefinition of the new coordinate $\tilde{x}$. At the left cold plate a potential should be of the order of $T_{-} / e$, hence the constant $B$ could be neglected in Eq. (6) provided $T_{+} \gg T_{-}$, Expressing the constant $A$ through the potential at the hot plate, one obtains 


$$
\varphi=\frac{\varphi_{+}-0.71 T_{+} / e}{\widetilde{L}_{x}} \widetilde{x}+0.71 T_{+} / e
$$

where

$$
\widetilde{L}_{x}=\int_{0}^{L_{x}} \frac{T_{+}^{3 / 2}}{T^{3 / 2}} d x
$$

The poloidal current is

$$
j_{x}=-\sigma_{\|} b_{x}^{2} \frac{T_{+}^{3 / 2}}{T^{3 / 2}} \frac{\varphi_{+}-0.71 T_{+} / e}{\widetilde{L}_{x}}
$$

The poloidal electron heat flow associated with the current according to Eq. (2) is

$$
q_{j}=-2.21 j_{x} T / e=+2.21 \sigma_{\|} b_{x}^{2} \frac{T_{+}^{3 / 2}}{T^{3 / 2}} \frac{\varphi_{+}-0.71 T_{+} / e}{e \widetilde{L}_{x}} T .
$$

The conductive poloidal electron heat flow is

$$
q_{\nabla T}=-\kappa b_{x}^{2} \frac{\partial T}{\partial x} .
$$

For the upstream it is possible to estimate ( $T_{u}$ is the upstream temperature)

$$
\widetilde{L}_{x}=\frac{T_{+}^{3 / 2}}{T^{3 / 2}} L_{x}, \quad \frac{\partial T_{u}}{\partial x} \sim \frac{T_{u}}{L_{x} / 2} .
$$

Taking $\varphi_{+} \sim 3 T_{+} / e$ close to the floating potential, one gets for the ratio of the upstream flows

$$
\frac{\left|q_{j}\right|}{\left|q_{\nabla T}\right|} \sim 1.7 \frac{T_{+}}{T_{u}}
$$


For example, if the ratio $T_{+} / T_{u}=0.2$ the convective electron heat flow is about $30 \%$ of the conductive heat flow. In reality, the electron heat flow to the colder divertor is smaller than to the hotter one, so one would expect larger effect for the inner colder divertor. Taking into account that the conductive part is transported to the plates both by ions and electrons while the convective flow is transported only by electrons, we have at the hot plate

$$
\frac{V_{e \|}-c_{s}}{c_{s}} \sim 1.7 \frac{T_{+}}{T_{u}} \frac{\gamma_{e}+\gamma_{i}}{\gamma_{e}},
$$

where $\gamma_{e}, \gamma_{i}$ are sheath transmission factors. So the additional electron velocity is comparable with the sound speed as is observed in the experiments and simulations.

\section{Conclusions}

The $\vec{E} \times \vec{B}$ poloidal drifts and parallel thermal currents are one of the sources of divertor asymmetries. The drifts directed away from the plate make the divertor denser and colder, while the drifts towards the plate lead to a decrease in density and increase in temperature. In the simulations performed for a CND symmetrical case this effect is observed both for HFS and LFS plates. In addition the thermal parallel current from the hotter to the colder divertor is responsible for the convective electron energy flow from the colder to the hotter divertor thus amplifying the asymmetry. The power up-down asymmetry observed in the MAST experiments might be connected with the convective electron energy flow caused by the thermal current. In the SND case with the normal direction of the magnetic field ( $\nabla B$ drift of ions towards the $X$-point) the initial HFS-LFS asymmetry is amplified both by the thermal current and the poloidal $\vec{E} \times \vec{B}$ drifts. The convective flow could be comparable with the conductive electron heat flow. Both thermal current and the poloidal $\vec{E} \times \vec{B}$ drifts could amplify considerably the existing asymmetry in the divertor plasma parameters.

\section{References}

1. P Stangeby, The plasma boundary of magnetic fusion devices, IOP publishing Ltd (2000)

2. V Rozhansky Contrib. Plasma Phys. 46 N7-9 (2006) 575

3. D Coster et al Proc. $32^{\text {nd }}$ EPS conf. on Plasma Phys. Tarragona 2005 P1.008

4. R Pitts et al J. Nucl. Mat. 337-339 (2005) 146

5. V Rozhansky et al J. Nucl. Mat. 313-316 (2003) 1141

6. V Rozhansky et al Nuclear Fus. 49 (2009) 025007 
7. P.J. Harbour Contrib. Plasma Phys. 28417 (1988)

8. V. Rozhansky, E. Kaveeva, S. Voskoboynikov et al Nuclear Fus. 43614 (2003)

9. Braginskii S.I. in Reviews of Plasma Physics, Vol. 1 (Leontovich M.A. Ed.), Consultants Bureau, New York. v. 1. 1965, p.205.

10. J-W Ahn et al $29^{\text {th }}$ Conf. on Plasma Phys. Contr. Fusion Montreaux (2002) ECA vol. 26B P.1-055

11. G De Temmerman Plasma Phys. Contr. Fus. 52 (2010) 095005

12. A.S. Kukushkin et al Nucl. Fusion 43 (2003) 716

\section{Figure captions}

Fig.1 Density (a), electron temperature (b) and electrostatic potential (c) for shot №17469.

Table 1. Shot №13018. Power through the separatrix Ptotal=2.0 Mw.

Table 2. Shot №17469. Ptotal=1.5 Mw.

Table 3. Shot №18751. Ptotal=1.8 Mw

Fig.2. The poloidal distribution of the parallel velocity at the LFS near the divertor plates for shot №13018: (a) outer upper divertor, $(b)$ outer lower divertor.

Fig.3. Poloidal density profiles at the LFS for shot №13018: $(a)$ outer upper divertor, $(b)$ outer lower divertor.

Fig.4. Scheme of the poloidal $\vec{E} \times \vec{B}$ drifts and the thermal currents in the SOL.

Fig.5. The saturation current at the divertor plates for shot №17469. (a) outer upper divertor $(b)$ outer lower divertor (c)

Fig.6. The electron temperature at the divertor plates for shot №17469. (a) outer upper divertor (b) outer lower divertor (c)

Fig.7. Poloidal electron heat flow between two flux surfaces (through the cell faces) in SOL for the low density case, 4-th cells from separatrix. Current contribution to the electron heat flow is switched off (black line) or switched on (red line).

Fig.8. Density distribution at the targets for the low density case: $(a)$-inner target, $(b)$-outer target.

Fig. 9. Electron temperature at the targets for the low density case: $(a)$-inner target, $(b)$-outer target.

Fig.10. Density distribution at the targets for the medium density case: $(a)$-inner target, $(b)$-outer target.

Fig.11. Electron temperature at the targets for the medium density case: $(a)$-inner target, $(b)$ outer target. 
Fig.12. Density $(a)$ and electron temperature $(b)$ distributions at the inner divertor for the high density case.

Fig.13. Scheme of the density distribution in the divertors in the presence of strong poloidal drifts. As a example $V_{x}^{E x B}=b_{x} c_{S}$ is assumed.

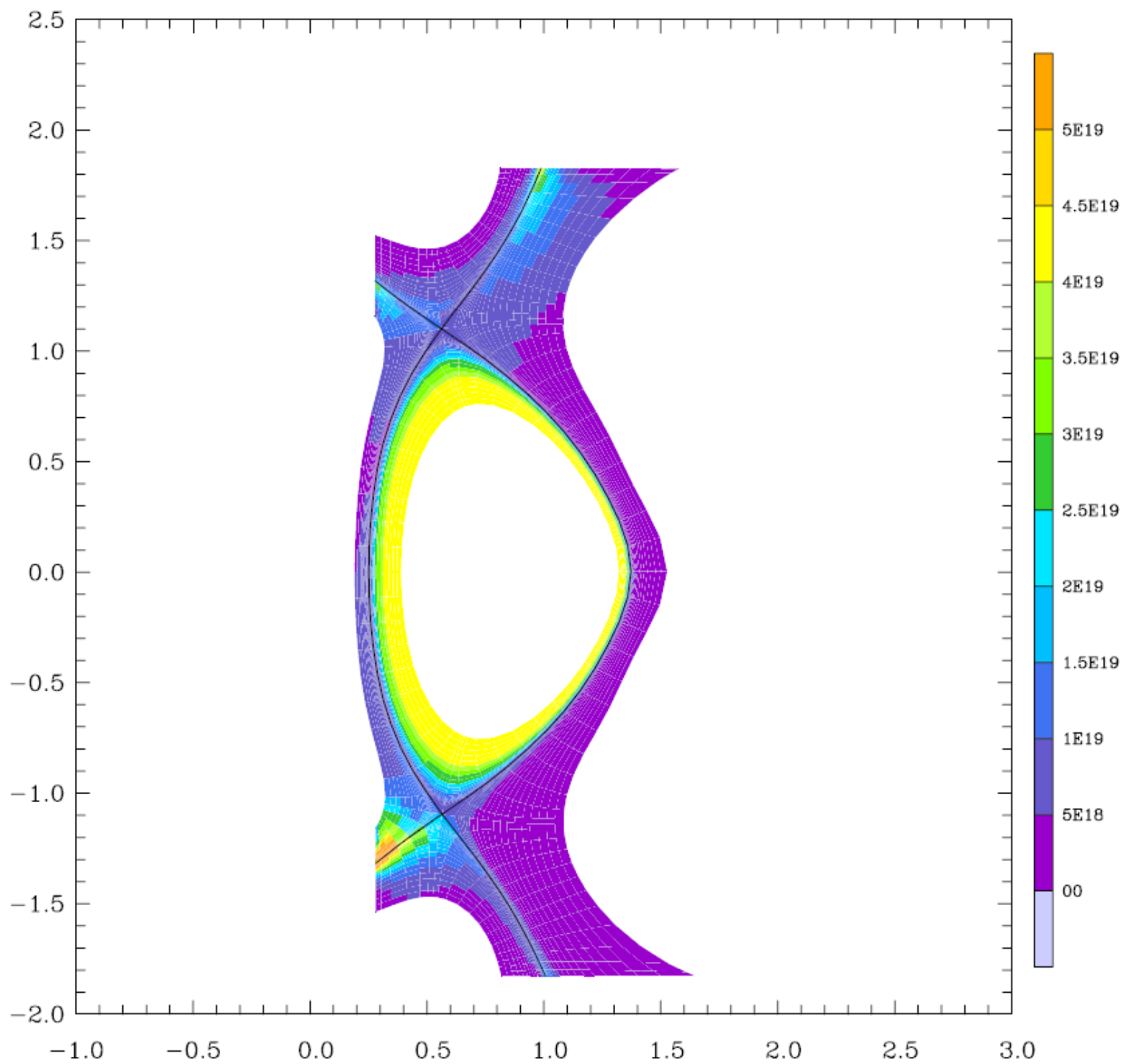

(a) 


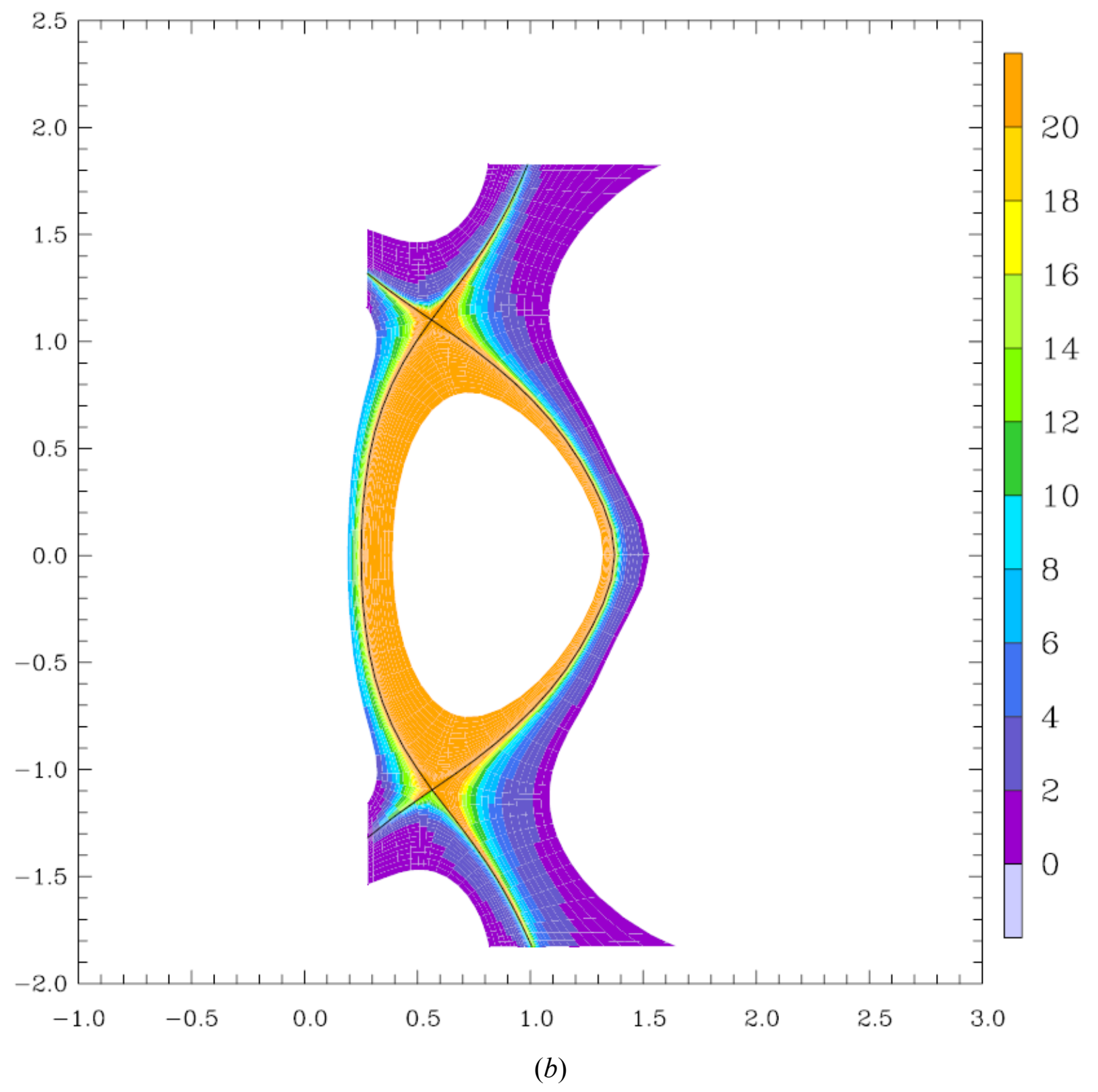




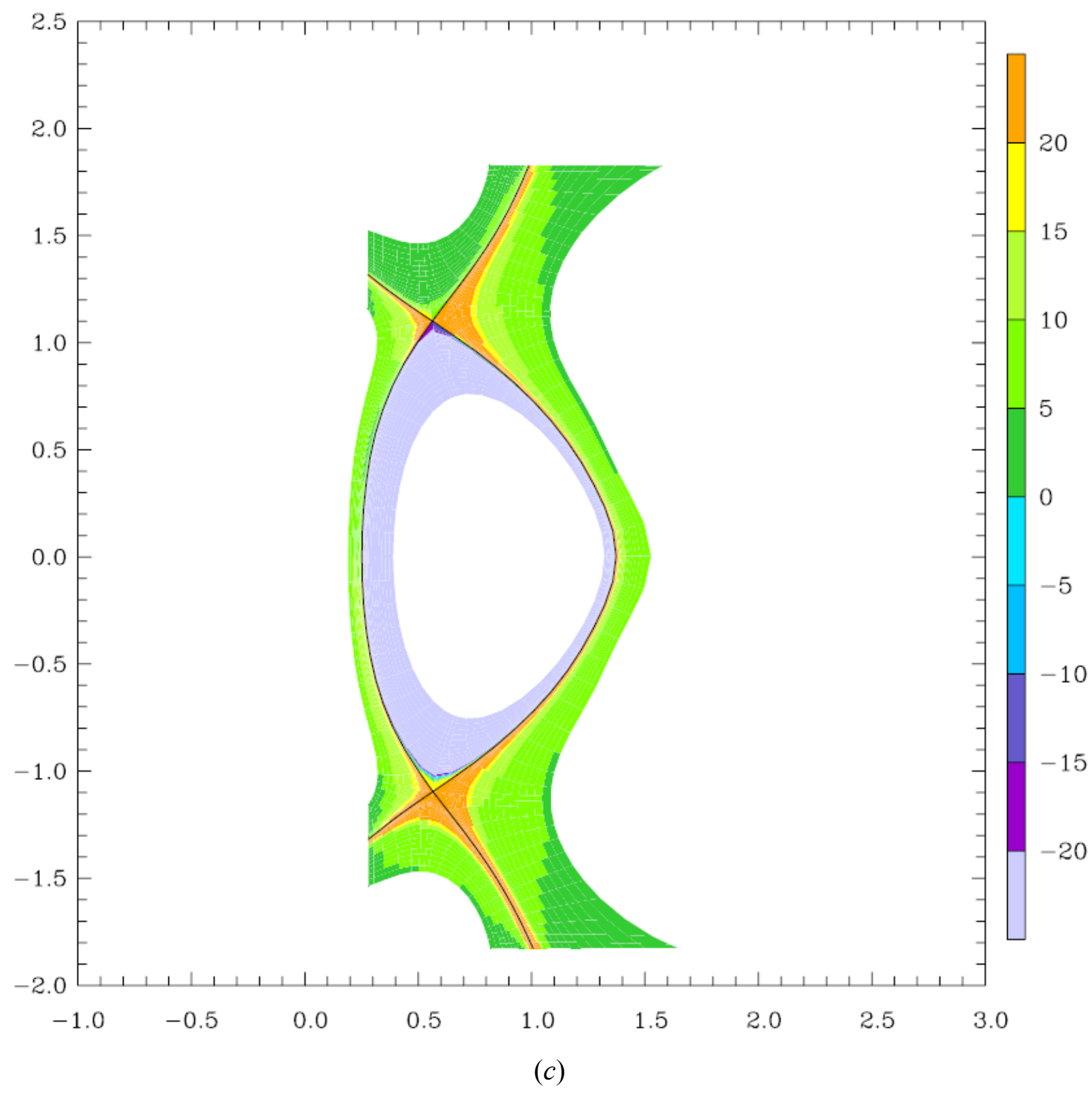

Fig.1 
Table .1

\begin{tabular}{|l|l|l|l|l|l|l|l|l|l|l|l|}
\hline Plate & $\begin{array}{l}T_{e} \\
e V\end{array}$ & $\begin{array}{l}T_{i} \\
e V\end{array}$ & $\begin{array}{l}n \\
m^{-3}\end{array}$ & $\begin{array}{l}j_{s a t} \\
\mathrm{kA} / \mathrm{m}^{2}\end{array}$ & $\begin{array}{l}p_{p l} \\
P a\end{array}$ & $\frac{e \varphi}{T_{e}}$ & $\begin{array}{l}P \\
M w\end{array}$ & $\begin{array}{l}V_{x}^{E x B} \\
\mathrm{~km} / \mathrm{s}\end{array}$ & $\begin{array}{l}V_{\|} \\
\mathrm{km} / \mathrm{s}\end{array}$ & $\begin{array}{l}n V_{x}^{E x B} \\
\mathrm{~s}^{-1} \mathrm{~m}^{-2}\end{array}$ & $\begin{array}{l}n c_{s} b_{x} \\
\mathrm{~s}^{-1} \mathrm{~m}^{-2}\end{array}$ \\
\hline OU & $\begin{array}{l}30 \\
(35)\end{array}$ & $\begin{array}{l}26 \\
(16)\end{array}$ & $\begin{array}{l}2.1 \cdot 10^{19} \\
\left(1.1 \cdot 10^{19}\right)\end{array}$ & $\begin{array}{l}32 \\
(18)\end{array}$ & $\begin{array}{l}173 \\
(98)\end{array}$ & 1.5 & 0.7 & 18 & 45 & $3.4 \cdot 10^{23}$ & $1.2 \cdot 10^{23}$ \\
\hline OL & $\begin{array}{l}44 \\
(37)\end{array}$ & $\begin{array}{l}22 \\
(15)\end{array}$ & $\begin{array}{l}0.8 \cdot 10^{19} \\
\left(1.1 \cdot 10^{19}\right)\end{array}$ & $\begin{array}{l}12 \\
(17)\end{array}$ & $\begin{array}{l}68 \\
(91)\end{array}$ & 0.9 & 0.5 & 6 & 59 & $3 \cdot 10^{22}$ & $7.6 \cdot 10^{22}$ \\
\hline IU & $\begin{array}{l}14 \\
(16)\end{array}$ & $\begin{array}{l}7 \\
(10)\end{array}$ & $\begin{array}{l}1.1 \cdot 10^{20} \\
\left(1.3 \cdot 10^{20}\right)\end{array}$ & $\begin{array}{l}41 \\
(46)\end{array}$ & $\begin{array}{l}237 \\
(287)\end{array}$ & 2.6 & 0.2 & 2 & 7 & $1.4 \cdot 10^{23}$ & $2.6 \cdot 10^{23}$ \\
\hline IL & $\begin{array}{l}15 \\
(15)\end{array}$ & $\begin{array}{l}38 \\
(7)\end{array}$ & $\begin{array}{l}1.2 \cdot 10^{20} \\
\left(1.4 \cdot 10^{20}\right)\end{array}$ & $\begin{array}{l}44 \\
(49)\end{array}$ & $\begin{array}{l}335 \\
(280)\end{array}$ & 2.8 & 0.2 & 0.5 & 15 & $2 \cdot 10^{23}$ & $2.7 \cdot 10^{23}$ \\
\hline
\end{tabular}

Table .2

\begin{tabular}{|l|l|l|l|l|l|l|l|l|l|l|l|}
\hline Plate & $\begin{array}{l}T_{e} \\
\mathrm{eV}\end{array}$ & $\begin{array}{l}T_{i} \\
\mathrm{eV}\end{array}$ & $\begin{array}{l}n \\
m^{-3}\end{array}$ & $\begin{array}{l}j_{s a t} \\
\mathrm{kA} / \mathrm{m}^{2}\end{array}$ & $\begin{array}{l}p_{p 1} \\
P a\end{array}$ & $\frac{e \varphi}{T_{e}}$ & $\begin{array}{l}P \\
M w\end{array}$ & $\begin{array}{l}V_{x}^{E x B} \\
\mathrm{~km} / \mathrm{s}\end{array}$ & $\begin{array}{l}V_{\|} \\
\mathrm{km} / \mathrm{s}\end{array}$ & $\begin{array}{l}n V_{x}^{E x B} \\
\mathrm{~s}^{-1} \mathrm{~m}^{-2}\end{array}$ & $\begin{array}{l}n c_{s} b_{x} \\
\mathrm{~s}^{-1} \mathrm{~m}^{-2}\end{array}$ \\
\hline OU & $\begin{array}{l}4 \\
(11)\end{array}$ & $\begin{array}{l}4.6 \cdot 10^{19} \\
(5.3)\end{array}$ & $\begin{array}{l}34 \\
\left(3.0 \cdot 10^{19}\right)\end{array}$ & $\begin{array}{l}83 \\
(27)\end{array}$ & 2.2 & 0.3 & 2 & 9 & $1 \cdot 10^{23}$ & $2.1 \cdot 10^{23}$ \\
\hline OL & $\begin{array}{l}17 \\
(13)\end{array}$ & $\begin{array}{l}8 \\
(5.7)\end{array}$ & $\begin{array}{l}1.7 \cdot 10^{19} \\
\left(2.7 \cdot 10^{19}\right)\end{array}$ & $\begin{array}{l}19 \\
(25)\end{array}$ & $\begin{array}{l}68 \\
(80)\end{array}$ & 1.5 & 0.4 & 8 & 21 & $1.5 \cdot 10^{23}$ & $1.2 \cdot 10^{23}$ \\
\hline IU & $\begin{array}{l}19 \\
(21)\end{array}$ & $\begin{array}{l}2.1 \cdot 10^{19} \\
(16)\end{array}$ & $\begin{array}{l}12 \\
\left(2.1 \cdot 10^{19}\right) \\
(16)\end{array}$ & $\begin{array}{l}73 \\
(118)\end{array}$ & 1.3 & 0.1 & 1 & 8 & $2.1 \cdot 10^{22}$ & $7.2 \cdot 10^{22}$ \\
\hline IL & $\begin{array}{l}7.4 \cdot 10^{19} \\
(18)\end{array}$ & $\begin{array}{l}4 \\
(13)\end{array}$ & $\begin{array}{l}\left.2.4 \cdot 10^{19}\right) \\
(17)\end{array}$ & $\begin{array}{l}91 \\
(115)\end{array}$ & 8.9 & 0.0 & 1 & 12 & $5.3 \cdot 10^{22}$ & $1.6 \cdot 10^{23}$ \\
\hline
\end{tabular}

Table .3

\begin{tabular}{|l|l|l|l|l|l|l|l|l|l|l|l|}
\hline Plate & $\begin{array}{l}T_{e} \\
e V\end{array}$ & $\begin{array}{l}T_{i} \\
e V\end{array}$ & $\begin{array}{l}n \\
m^{-3}\end{array}$ & $\begin{array}{l}j_{s a t} \\
k A / m^{2}\end{array}$ & $\begin{array}{l}p_{p 1} \\
P a\end{array}$ & $\frac{e \varphi}{T_{e}}$ & $\begin{array}{l}P \\
M w\end{array}$ & $\begin{array}{l}V_{x}^{E x B} \\
k m / s\end{array}$ & $\begin{array}{l}V_{\|} \\
k m / s\end{array}$ & $\begin{array}{l}n V_{x}^{E x B} \\
s^{-1} m^{-2}\end{array}$ & $\begin{array}{l}n c_{s} b_{x} \\
s^{-1} m^{-2}\end{array}$ \\
\hline OU & $\begin{array}{l}11 \\
(24)\end{array}$ & $\begin{array}{l}5 \\
(8)\end{array}$ & $\begin{array}{l}4.8 \cdot 10^{19} \\
\left(1.6^{19} \cdot 10^{19}\right)\end{array}$ & $\begin{array}{l}42 \\
(20)\end{array}$ & $\begin{array}{l}123 \\
(84)\end{array}$ & 2.5 & 0.4 & 4 & 12 & $2 \cdot 10^{23}$ & $2.6 \cdot 10^{23}$ \\
\hline OL & $\begin{array}{l}25 \\
(26)\end{array}$ & $\begin{array}{l}10 \\
(8)\end{array}$ & $\begin{array}{l}1.4 \cdot 10^{19} \\
\left(1.5 \cdot 10^{19}\right)\end{array}$ & $\begin{array}{l}18 \\
(19)\end{array}$ & $\begin{array}{l}78 \\
(80)\end{array}$ & 1.2 & 0.5 & 9 & 26 & $1.3 \cdot 10^{23}$ & $1.1 \cdot 10^{23}$ \\
\hline IU & $\begin{array}{l}30 \\
(48)\end{array}$ & $\begin{array}{l}9 \\
(37)\end{array}$ & $\begin{array}{l}1.5 \cdot 10^{19} \\
\left(0.8 \cdot 10^{19}\right)\end{array}$ & $\begin{array}{l}10 \\
(9)\end{array}$ & $\begin{array}{l}73 \\
(103)\end{array}$ & 1.0 & 0.1 & 1.5 & 10 & $2.1 \cdot 10^{22}$ & $5.9 \cdot 10^{22}$ \\
\hline IL & $\begin{array}{l}8 \\
(45)\end{array}$ & $\begin{array}{l}10 \\
(33)\end{array}$ & $\begin{array}{l}4.5 \cdot 10^{19} \\
\left(0.8 \cdot 10^{19}\right)\end{array}$ & $\begin{array}{l}24 \\
(9)\end{array}$ & $\begin{array}{l}126 \\
(100)\end{array}$ & 3.7 & 0.0 & 1 & 13 & $2.2 \cdot 10^{23}$ & $1.5 \cdot 10^{23}$ \\
\hline
\end{tabular}




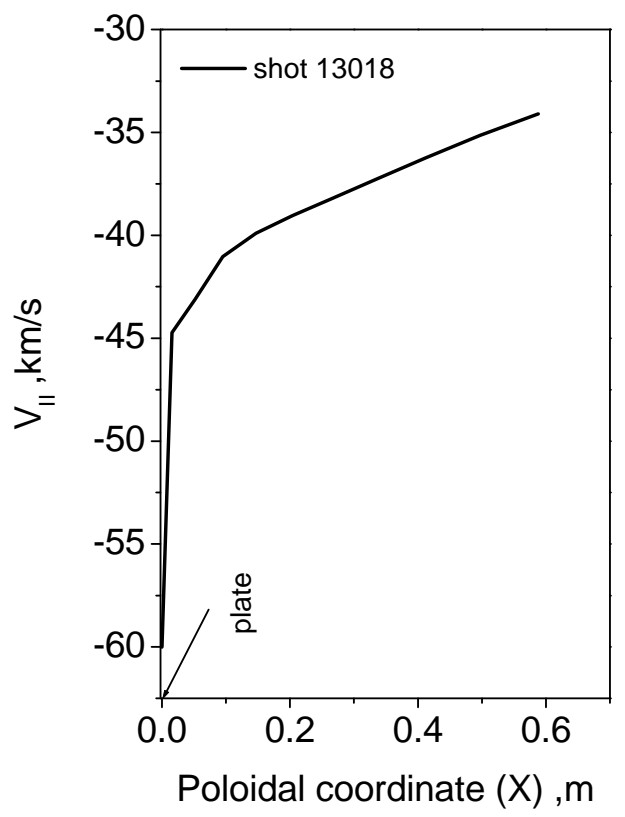

(a)

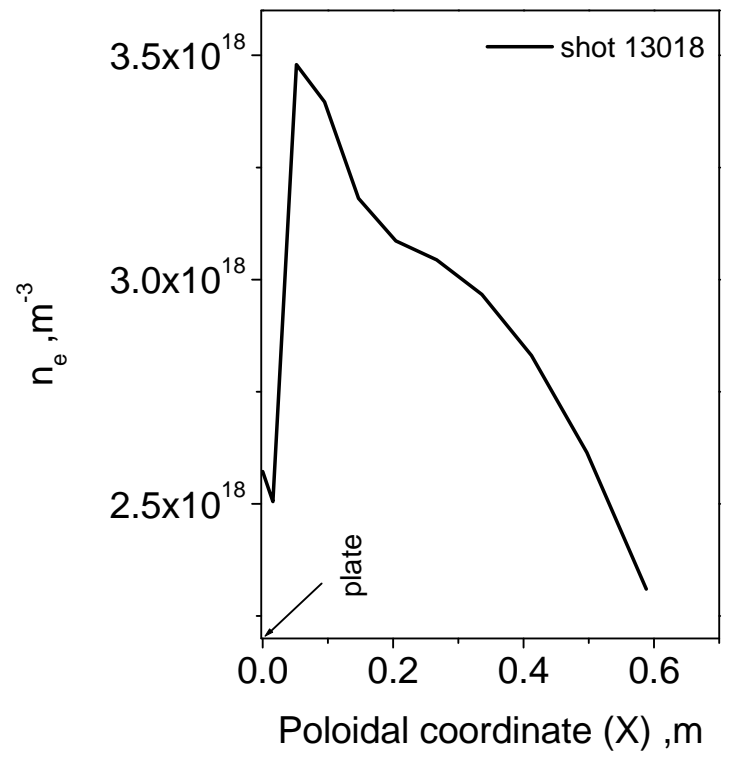

(a)

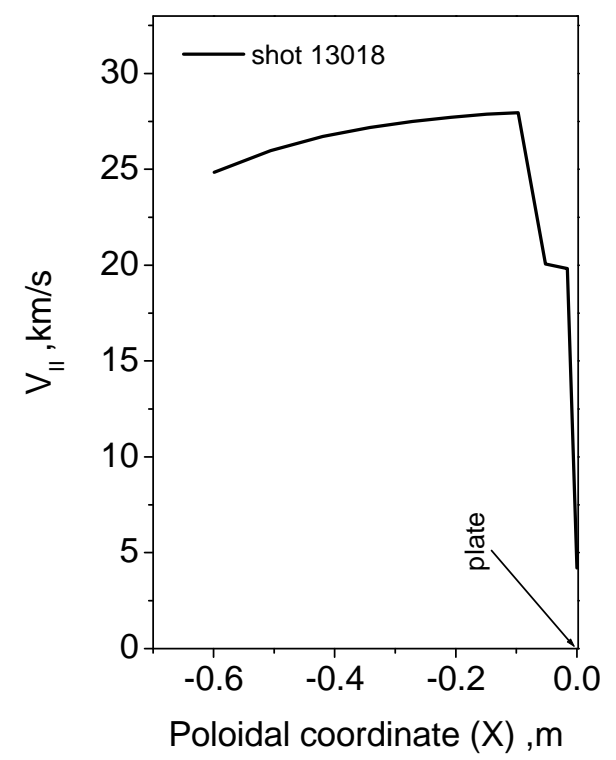

(b)

Fig.2

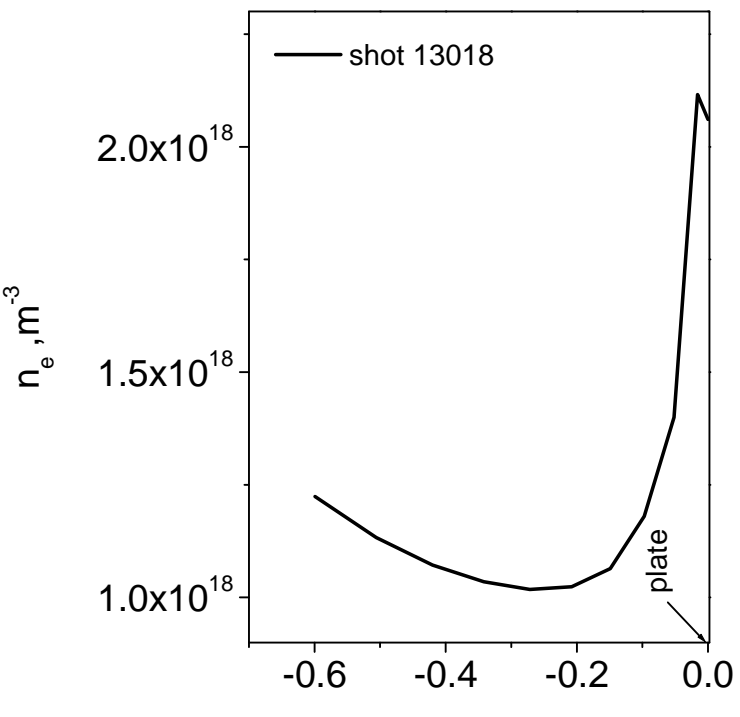

Poloidal coordinate $(X), \mathrm{m}$

(b)

Fig.3 


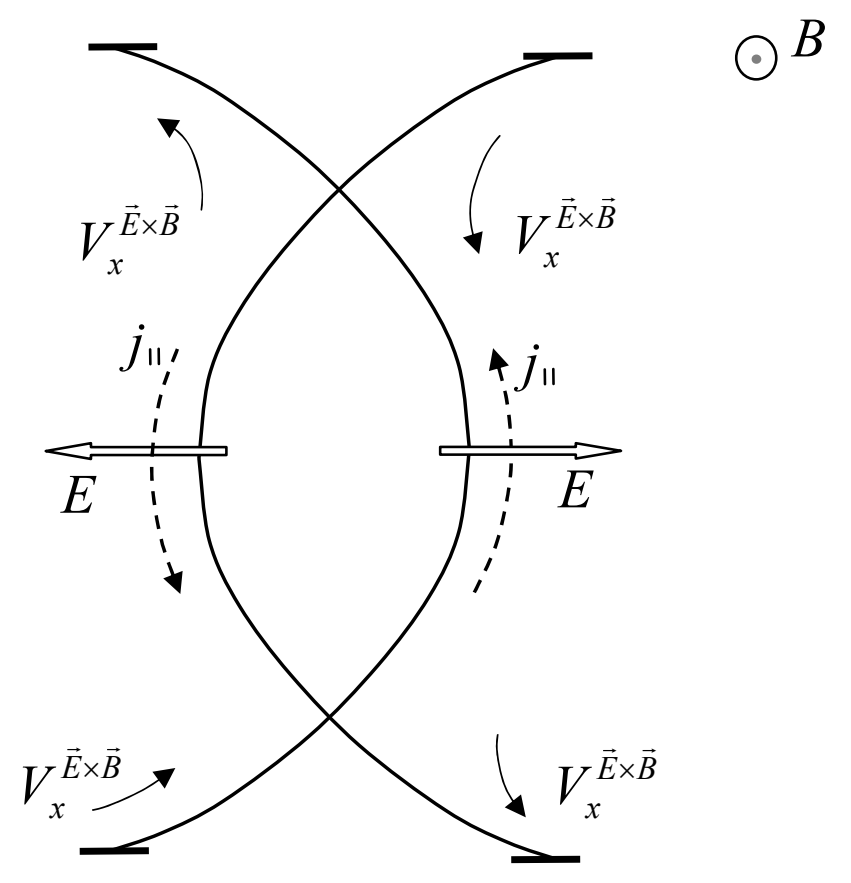

Fig.4. 


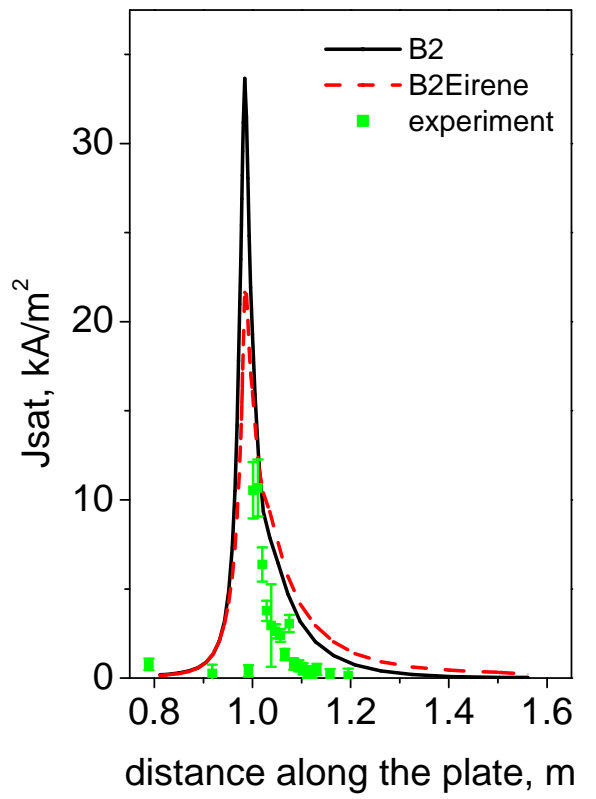

(a)

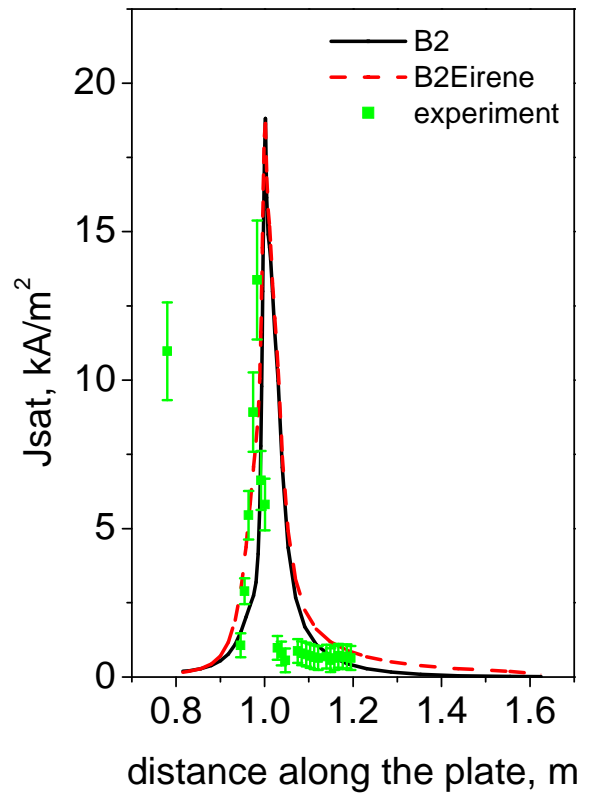

(b)

Fig. 5. 


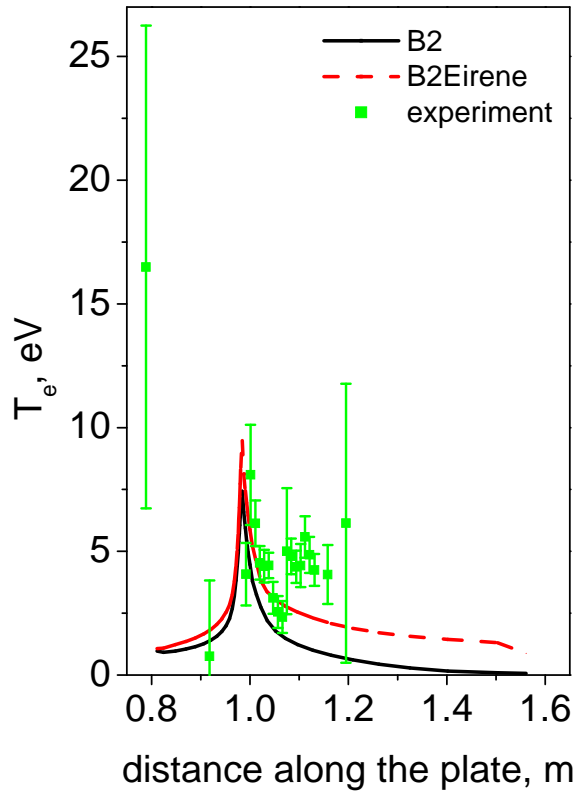

(a)

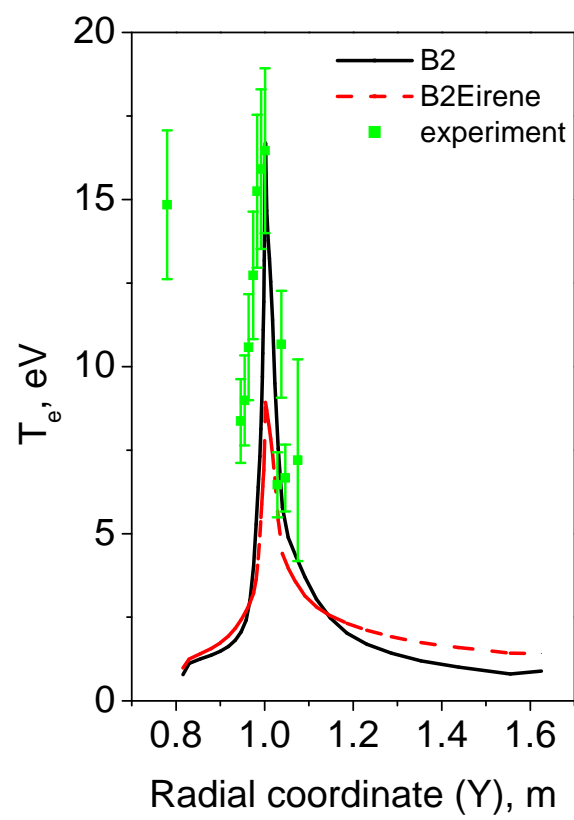

(b)

Fig. 6 


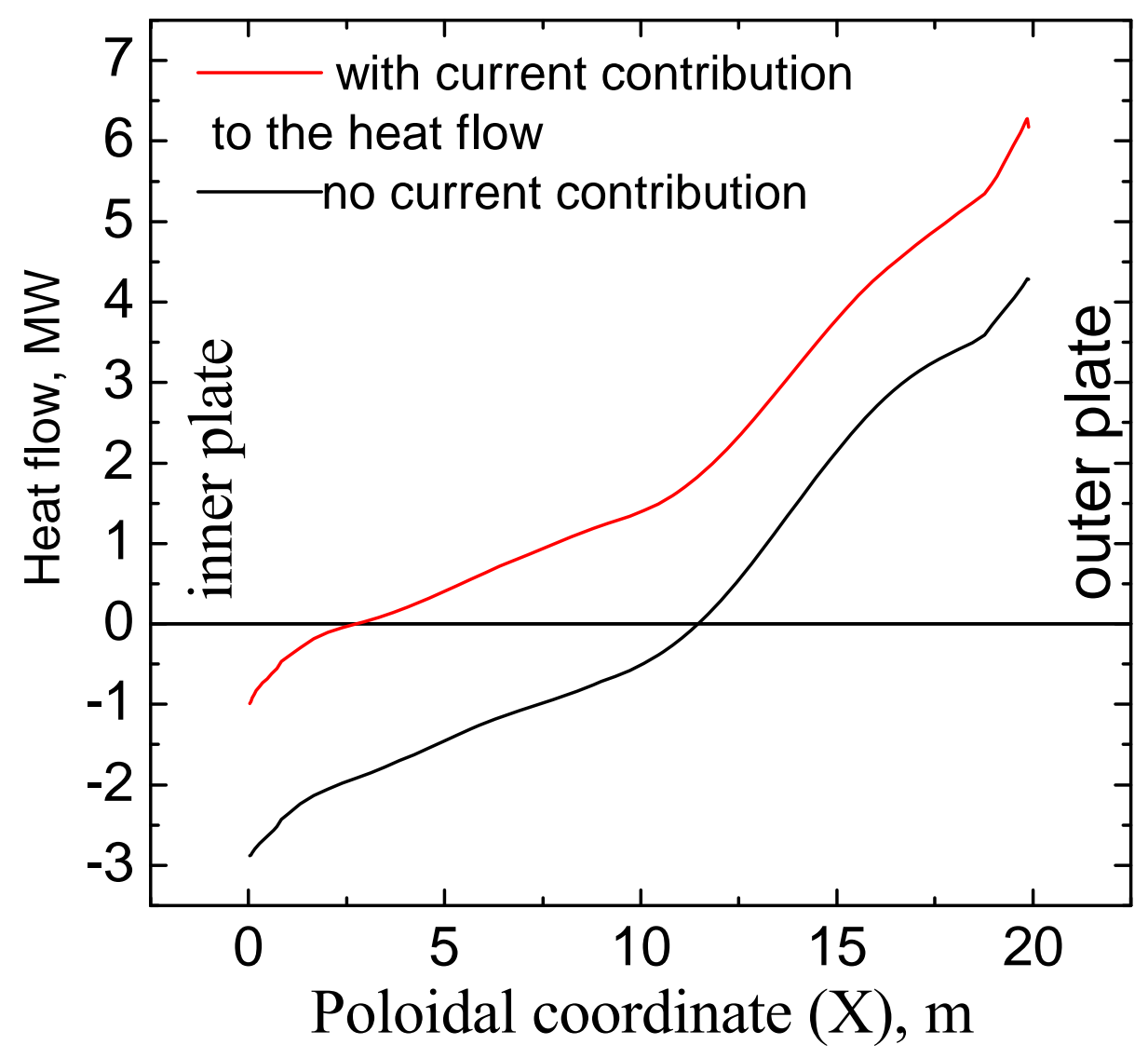

Fig.5 


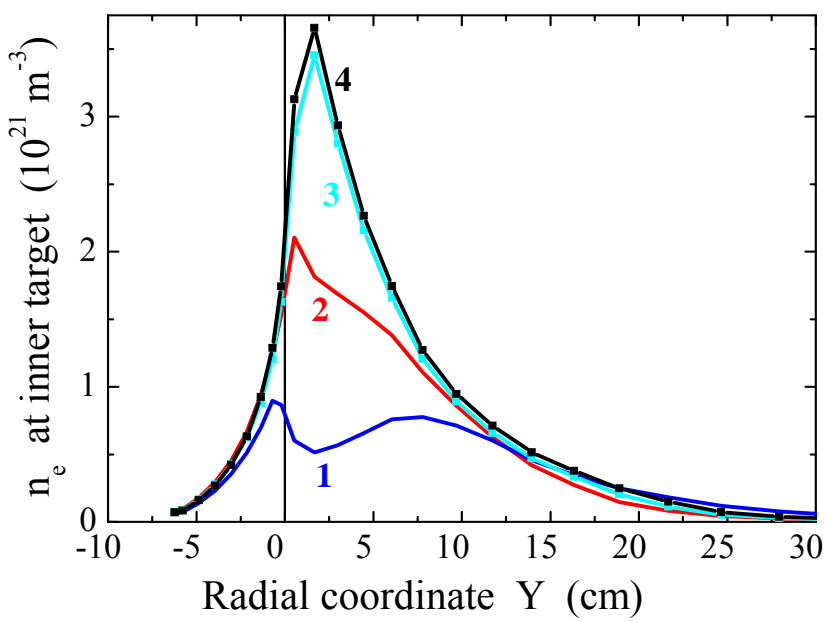

(a)

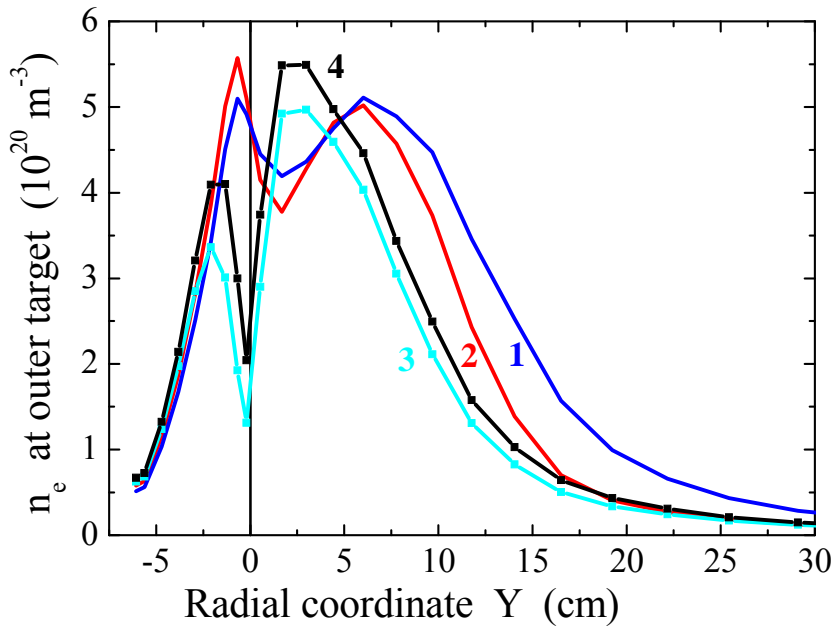

(b)

Fig.6

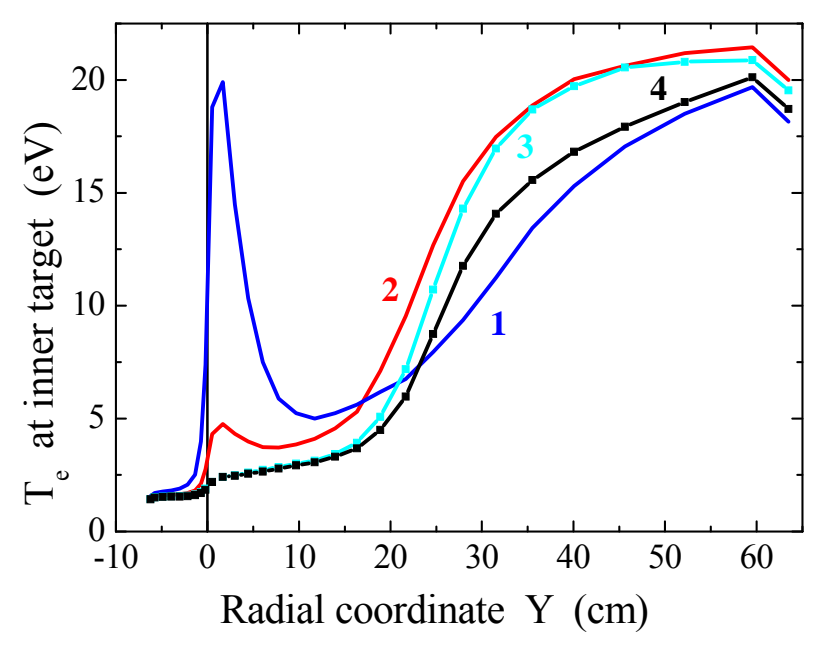

(a)

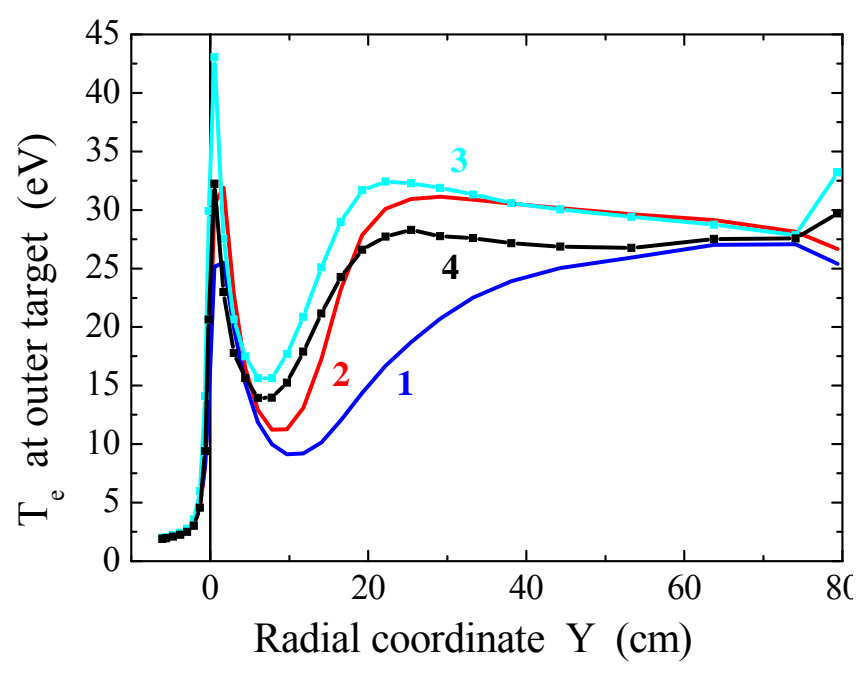

(b)

Fig.7 


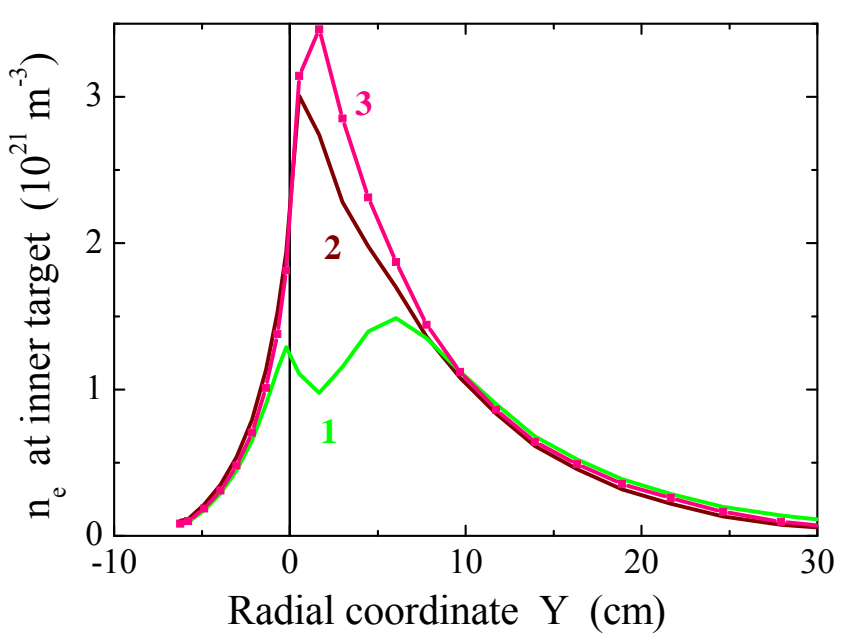

(a)

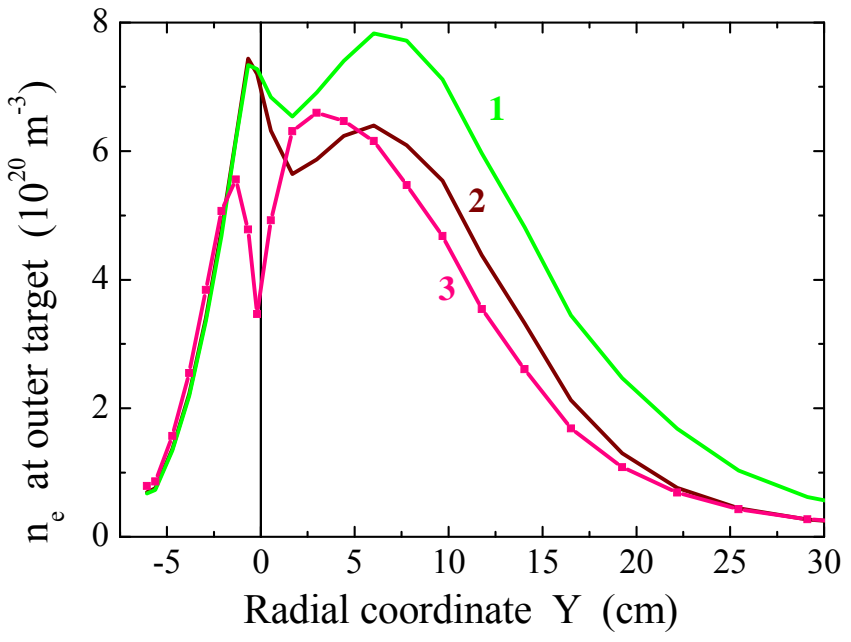

(b)

Fig.8

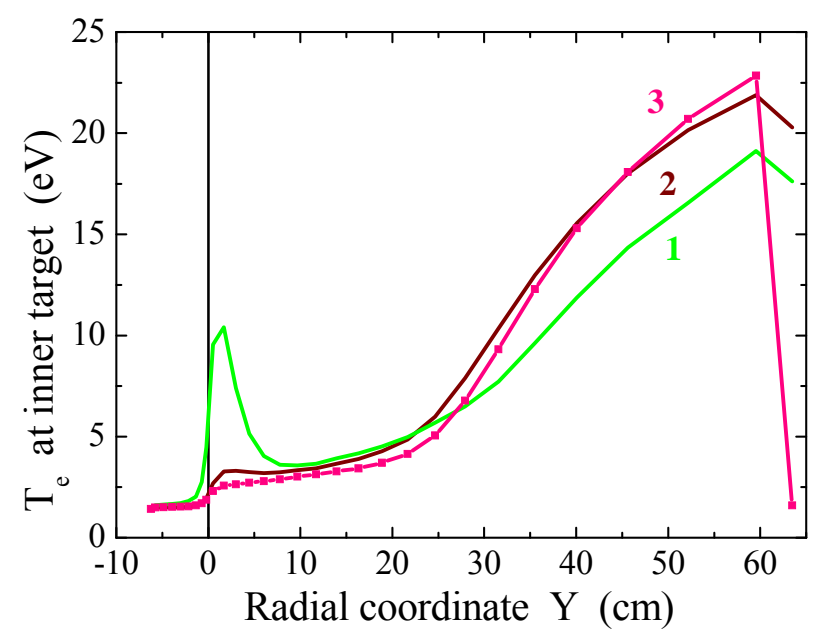

(a)

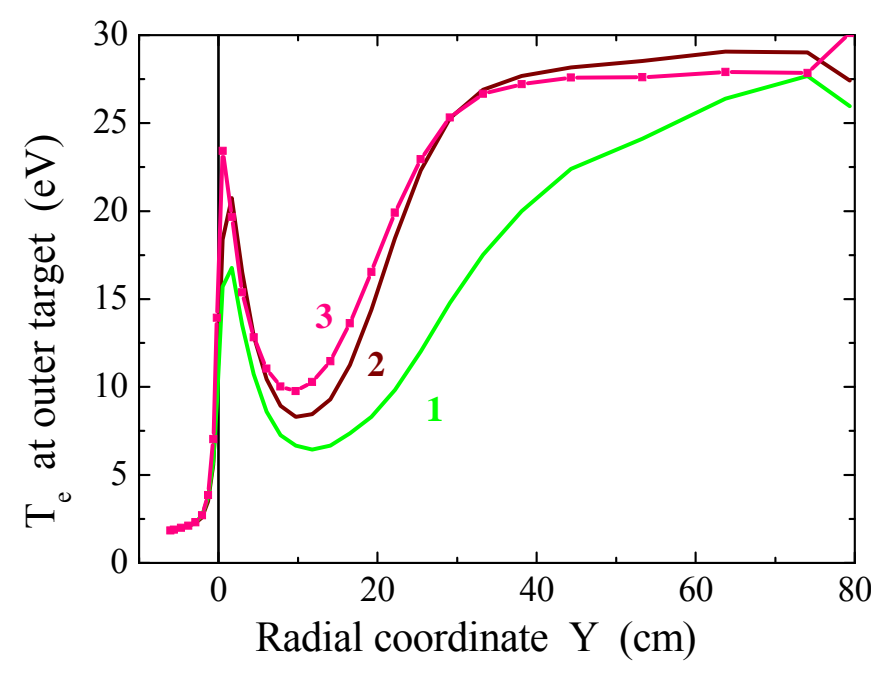

(b)

Fig.9 


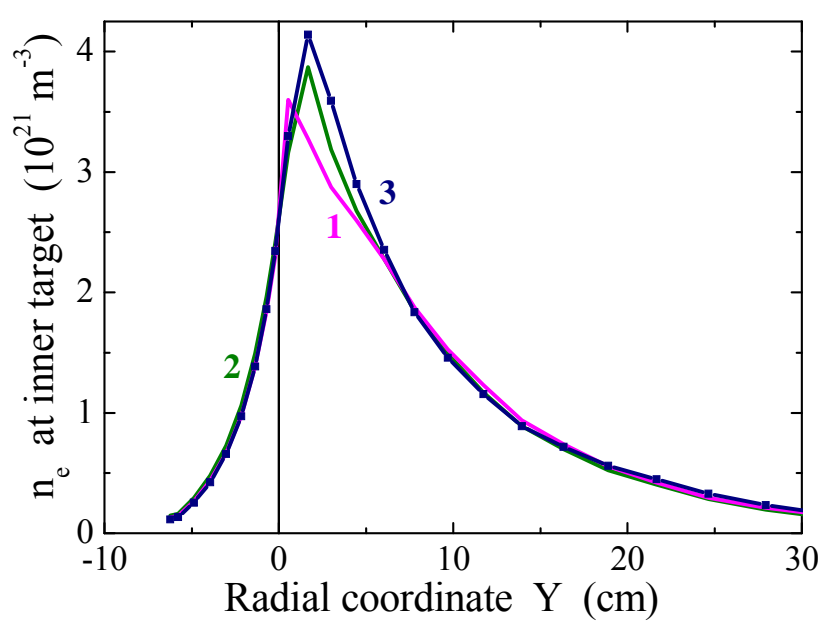

(a)

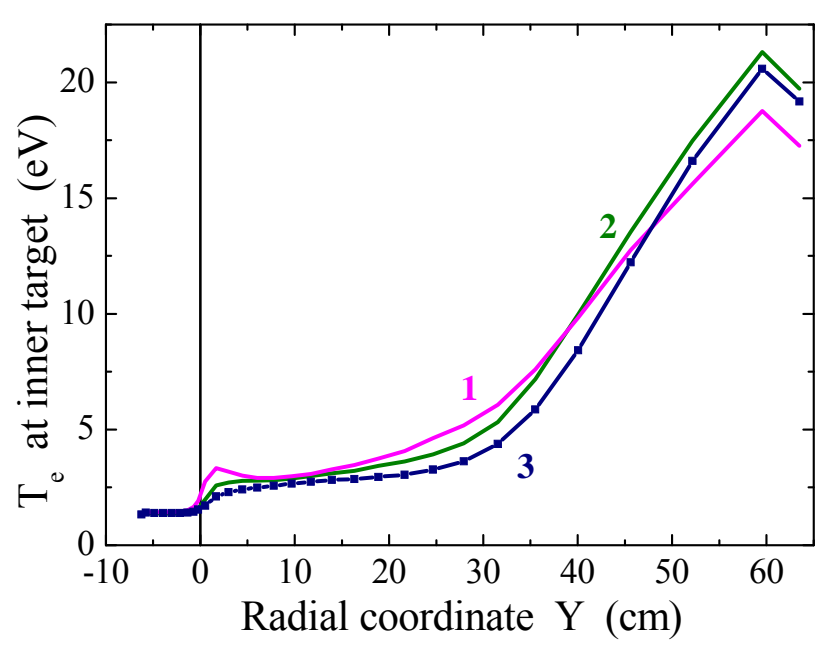

(b)

Fig.10 


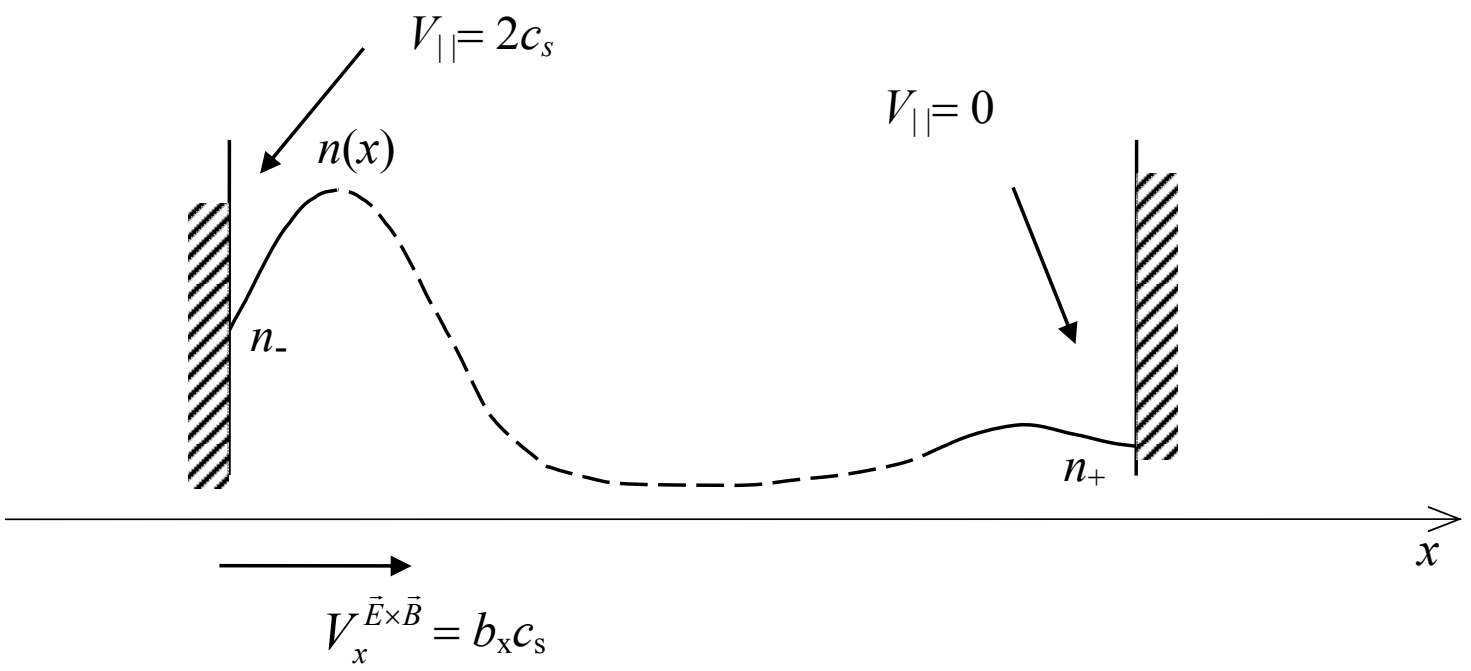

Fig.11 